\title{
Personalized genomic medicine: non-small-cell lung cancer and anaplastic lymphoma kinase
}

\author{
Umberto Malapelle ${ }^{1}$, Luigi Della Gravara², Ciro Battiloro ${ }^{3}$, Aniello Avellino ${ }^{3}$, Danilo Rocco ${ }^{3}$ \\ 'Department of Public Health, "Federico II" University, Naples 80131, Italy. \\ 2Department of Precision Medicine, “Luigi Vanvitelli" University, Naples 80131, Italy. \\ ${ }^{3}$ Department of Pulmonary Oncology, AORN dei Colli Monaldi, Naples 80131, Italy. \\ Correspondence to: Dr. Danilo Rocco, Department of Pulmonary Oncology, AORN dei Colli Monaldi, Naples 80131, Italy. \\ E-mail: danilorocc@yahoo.it
}

\begin{abstract}
How to cite this article: Rocco D, Della Gravara L, Battiloro C, Avellino A, Malapelle U. Personalized genomic medicine: non-small-cell lung cancer and anaplastic lymphoma kinase. J Trans/ Genet Genom 2019;3:3. https://doi.org/10.20517/jtgg.2018.29
\end{abstract}

Received: 21 Sep 2018 First Decision: 11 Dec 2018 Revised: 20 Dec 2018 Accepted: 21 Dec 2018 Published: 19 Feb 2019

Science Editor: Sheng-Ying Qin Copy Editor: Cui Yu Production Editor: Huan-Liang Wu

\begin{abstract}
Genomic medicine, that is to say, using genomic information about a patient in order to set the diagnostic path and to tailor therapy to his/her specific characteristics, is one of the cornerstones of modern precision medicine and forms an integral part of several fields, oncology first of all. Lung cancer is the leading cause of cancer mortality, causing more than 1.6 million deaths worldwide per year and non-small-cell lung cancer (NSCLC) accounts for approximately $85 \%$ of lung cancers. In a small subset of NSCLC ( $5 \%-8 \%$ ), we can detect a genomic rearrangement on chromosome 2 , between the Echinoderm microtubule-associated protein-like 4 (EMLA) gene and the anaplastic lymphoma kinase ( $A L K)$ gene, resulting in the chimeric protein EML4-ALK, that acts as an oncogene and that can be specifically targeted by an ALKtyrosine kinase inhibitor (TKI) therapy. However, a major clinical challenge is represented by the fact that, after a first line ALK-TKI treatment, patients eventually develop acquired resistance to these agents, opening new scenarios for the right second-line drug choice.
\end{abstract}

Keywords: Precision medicine, genomic medicine, non-small-cell lung cancer, Echinoderm microtubule-associated proteinlike 4-anaplastic lymphoma kinase, anaplastic lymphoma kinase, tyrosine kinase inhibitor

\section{INTRODUCTION}

Targeted therapy is one of the greatest achievements in modern day precision oncology, allowing to specifically target molecular drivers, mainly responsible for cancer-cells development, proliferation and survival in several tumor subsets, anaplastic lymphoma kinase (ALK) rearranged non-small-cell lung cancer (NSCLC) included.

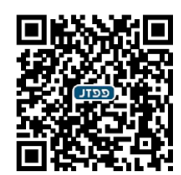




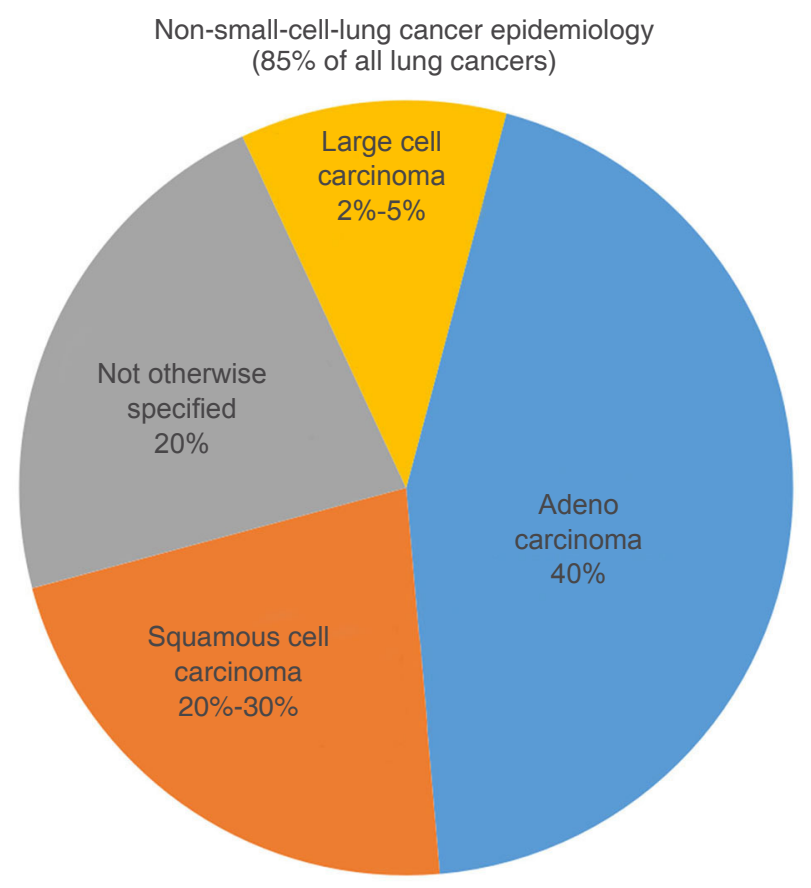

Figure 1. Non-small-cell-lung cancer epidemiology

With reference to this particular cancer subtype, while treatment with ALK-tyrosine kinase inhibitor (TKI) gives raise to no concerns and is currently considered the standard-of-care by international guidelines, acquired resistance mechanisms to these agents represent a major clinical challenge. In fact, not only we do not fully understand the mechanism behind this phenomenon, but we are also struggling to develop effective drugs against the known resistance pathways.

Therefore, this paper aims to provide an up-to-date state-of-the-art review about ALK + NSCLC, genomics, epidemiology, diagnosis, treatment and acquired resistance mechanisms, jointly with an analysis about future developments and directions in this field.

\section{NSCLC EPIDEMIOLOGY}

Currently, lung cancer is both the most diagnosed and the deadliest cancer worldwide, and NSCLC accounts for $85 \%$ of all cases. However, NSCLC is not one single entity, in fact, it is subdivided into adenocarcinoma also known as lung adenocarcinoma (AC and LUAD respectively, 40\% of all NSCLCs), lung squamous cell carcinoma (or LUSC, $20 \%-30 \%$ of all NSCLCs ), large cell carcinoma (2\%-5\% of all NSCLCs) or not otherwise specified (20\% of all NSCLCs), according to the histological type [Figure 1]; and in wild-type (without any known mutation) or mutated ("oncogene addicted"), if a mutation is present.

Presently, we are only able to specifically target oncogenic mutations in the adenocarcinoma histological type, most notably epidermal growth factor receptor (EGFR, 15\%-20\% of all AC NSCLC) and ALK (4\%-6\% of all AC NSCLC, mainly younger and non smokers/light smokers patients $)^{[1-6]}$.

\section{THE ALK GENE}

The $A L K$ gene, located on the short arm of chromosome 2 (2p23), encodes for the homonymous receptor tyrosine kinase (ALK-RTK), consisting of an extracellular, a transmembrane and a catalytic cytoplasmic portion (that harbors the ATP binding cleft, responsible for starting phosphorylation and thus signal transduction) ${ }^{[7]}$. 
Physiologically, ALK-RTK expression is limited to rare embryonic and adult brain tissue cells, suggesting a role in neural development and function ${ }^{[8,9]}$. However, in a small subset of NSCLC affected patients (4\%-6\%), typically following an inversion rearrangement in the short arm of chromosome 2 between the ALK gene and the Echinoderm microtubule-associated protein-like 4 (EML4) gene [inv2(p21;p23)], the resulting chimeric fusion protein (EML4-ALK) becomes aberrantly expressed, resulting in enhanced tyrosine kinase activity and constitutive activation of the signaling pathways mediated by mitogen-activated protein kinase, Janus kinase-signal transducer and activator of transcription proteins (JAK-STAT), Phosphatidylinositol4,5-bisphosphate 3-kinase-RAC-alpha serine/threonine-protein kinase, leading to cell proliferation and anti-apoptosis and eventually to tumorigenesis ${ }^{[10-12]}$; on a side note, ALK rearrangements with other genes (fusion partners) other than EML4 have also been reported (e.g., TFG, KLC1, PTPN3). However, the eventual outcome does not vary ${ }^{[13]}$.

Moreover, based on the EML4 breakpoint, we can further identify several EML4-ALK fusion variants (15 to date), expressing different amounts of EML tandem atypical $\beta$-propeller in EML protein (TAPE) domain, or no TAPE domain at all; among all EML4-ALK fusion variants, variants 1 (partial TAPE), 2 (partial TAPE) and $3 \mathrm{a} / \mathrm{b}$ (no TAPE) are the most common ones, accounting for approximately $90 \%$ of all cases. According to the most recent studies, variants lacking TAPE domain show earlier TKI-treatment failure and worse outcome, when compared to TAPE-harboring ones ${ }^{[14,15]}$.

\section{DIAGNOSING ALK + NSCLC}

Currently, we can use four different methods to detect ALK rearrangements: fluorescent in situ hybridization (FISH), immunohistochemistry (IHC), reverse transcriptase polymerase chain reaction (RT-PCR) and next generation sequencing (NGS); FISH and IHC are the only US Food and Drug Administration (FDA) approved techniques ${ }^{[16,17]}$.

\section{FISH}

To date, FISH is considered the gold standard assay for diagnosing ALK rearrangements. The rationale behind this method is represented by the fact that using two differently colored break-apart probes (green and red) specific to the inversion breakpoints of the $A L K$ gene, we will obtain a single yellow signal in non rearranged cells, while two split signals (green and red) will be obtained in rearranged cells; however, although very reliable, this technique cannot be automated and requires trained personnel and equipment (fluorescence microscope and the devices for the hybridization probes) for results interpretation ${ }^{[16,17]}$.

\section{IHC}

Whereas ALK rearrangements are followed by EML4-ALK expression, IHC relies on highly ALK-specific monoclonal antibodies to detect the products of this inversion on formalin fixed tissue sections; while lowcost and easy to perform, IHC requires laboratory validation and standardization ${ }^{[16,17]}$.

\section{RT-PCR}

The use of this method is currently not recommended, in fact, it does not provide a good quality of RNA from the formalin-fixed paraffin embedded tissue and furthermore can only detect known fusion partners. However, with this approach subjectivity in assessment of the analysis can be completely ruled out, unlike IHC and FISH ${ }^{[16,17]}$.

\section{NGS}

Even though NGS is still not FDA-approved, it is an undoubtedly promising technique, in fact, it grants screening of both known and novel ALK gene rearrangements, alongside with screening for other NSCLCrelated gene mutations; nevertheless, trained personnel is required for results interpretation and costs are still prohibitive ${ }^{[16,18]}$. 


\section{ALK + NSCLC TREATMENT: ALK TKI}

Being able to incorporate genomic information into the diagnostic and clinical pathways, shaping personalized precision cancer therapies, has been one of the biggest shift in modern day oncology, and ALK + NSCLC treatment is a perfect example. In fact, according to the most recent American Society of Clinical Oncology and the European Society for Medical Oncology guidelines, ALK-TKIs presently represent the standard of care in the treatment of this subgroup of patients, granting results far beyond chemotherapy; more specifically, three ALK-TKIs (alectinib, ceritinib and crizotinib) are FDA and European Medicines Agency approved for the clinical practice, while another TKI (brigatinib) is only FDA-approved for the clinical practice ${ }^{[19-21]}$. Furthermore, all of the ALK-TKIs share the same mechanism of action: specifically and competitively binding the ATP binding pocket they manage to block phosphorylation, thus inhibiting TKI signal transduction pathways and ultimately cell survival and proliferation ${ }^{[22]}$.

However, when compared to first-generation ALK-TKIs (crizotinib), second generation ones (ceritinib, alectinib and brigatinib) manage to grant superior clinical performances thanks to the improved chemical structure, specifically designed in order to be more selective and potent (and thus associated with lower IC50) and to be able to easily cross the blood-brain barrier, due to the high rates of brain metastases in ALK + NSCLCs ${ }^{[23,24]}$.

\section{First-line ALK-TKI}

\section{Crizotinib}

Crizotinib was the first FDA-approved ALK-TKI, receiving accelerated approval in 2011 and regular approval in 2013 for ALK + NSCLC affected patients, based on the findings from the A8081007 study, a randomized (1:1) trial in which 347 ALK + NSCLC affected patients that had already received a previous platinumbased treatment were randomized to receive either crizotinib or standard of care chemotherapy (docetaxel/ pemetrexed). Results favored crizotinib over chemotherapy: progression free survival (PFS): 7.7 months vs. 3.0 months, respectively, plus a $46 \%$ absolute increase in objective response rate $(\mathrm{ORR})^{[25]}$.

These promising results were then confirmed in a first line setting by the PROFILE 1,014 study, in which 343 naive ALK + NSCLC affected patients were randomized to receive crizotinib or standard of care chemotherapy (cisplatin/carboplatin + pemetrexed). In fact, results were completely in favor of the crizotinib arm: PFS: 10.9 months (crizotinib) vs. 7.0 months (chemotherapy), ORR: 64\% (crizotinib) vs. $45 \%$ (chemotherapy), OS: not reached (NR) (crizotinib) (95\% CI, 45.8 months to NR) vs. 47.5 months (chemotherapy), survival probability at 4 years: $56.6 \%$ (crizotinib) vs. $49.1 \%$ (chemotherapy) ${ }^{[26,27]}$.

\section{Ceritinib}

Ceritinib was the second ALK-TKI to receive FDA approval for the treatment of naive ALK + NSCLC patients in 2017, thanks to the results coming from the ASCEND 4 study, a phase III trial randomizing 376 patients (1:1) to receive first line ceritinib or standard of care chemotherapy (cisplatin/carboplatin + pemetrexed). The ceritinib regimen performed significantly better than the platinum-based one according to every endpoint: PFS: 16.6 months (ceritinib) vs. 8.1 months (chemotherapy), ORR: 73\% (ceritinib) vs. $27 \%$ (chemotherapy); moreover, unlike crizotinib, ceritinib performed greatly also with respect to central nervous system (CNS) lesions, with an overall intracranial response rate of 57\% vs. 22\% (chemotherapy), and a median CNS response duration of 16.6 months ${ }^{[28]}$.

\section{Alectinib}

Alectinib was the third FDA-approved ALK-TKI (2017) for the treatment of naive NSCLC harboring ALK rearrangements in the first line setting and is currently considered the best upfront treatment available, due to the findings coming from the ALEX trial. 
In this phase III trial, 303 naive ALK + NSCLC patients were randomized (1:1) to receive upfront alectinib or crizotinib and the alectinib arm achieved consistently better results: ORR: $82.9 \%$ (alectinib) vs. $75.5 \%$ (crizotinib), duration of response (DOR): 33.3 months (alectinib) vs. 11.1 months (crizotinib), PFS: 34.8 months (alectinib) vs. 10.9 months (crizotinib) ${ }^{[29]}$.

\section{Second-line ALK-TKI}

To date, ceritinib, alectinib and brigatinib are FDA-approved for the second-line treatment of ALK + NSCLC patients, after intolerance to or failure of a first line crizotinib treatment.

\section{Ceritinib}

In 2014, ceritinib was the first ALK-TKI to be granted FDA approval for the treatment of ALK + NSCLC patients who had progressed on or were intolerant to crizotinib treatment, thanks to a single-arm trial (163 patients) that showed an ORR of $44 \%$ and a DOR of 7.1 months $^{[30]}$.

\section{Alectinib}

In 2015, alectinib was granted FDA-approval for the treatment of ALK + NSCLC patients, after intolerance to or failure of a first line crizotinib treatment, based on the findings from two different single-arm trials, that respectively showed an ORR of $38 \%$ and $44 \%$, a DOR of 7.5 months and 11.2 months, alongside with a CNS ORR of $61 \%$ and a CNS DOR of 9.1 months $^{[31]}$.

\section{Brigatinib}

In 2017, brigatinib was granted FDA approval for the treatment of ALK + NSCLC patients who had progressed on or were intolerant to crizotinib treatment, based on the results from the ALTA trial, a randomized phase II trial, evaluating two regimens of brigatinib $90 \mathrm{mg} v s .180 \mathrm{mg}$.

Two hundred and twenty-two patients were randomized (1:1) and the results showed: ORR: $48 \%$ vs. 53\%, CNS ORR: $42 \%$ vs. $63 \%$, respectively, plus a DOR of 13.8 months and a CNS DOR of 5.6 months (180 mg regimen ${ }^{[32]}$.

\section{ACQUIRED RESISTANCE}

Regarding ALK-TKI treatment, the main hurdle to overcome is represented by the cancer cells acquired mechanisms of resistance to therapy, that ultimately lead to the progression of disease; even though these mechanisms can consistently vary according to the administered ALK-TKI, clinical and pre-clinical models indicate that resistance can develop through 3 main mechanisms: activation of other oncogenic signals that allow the tumor to bypass the ALK signaling pathway, TKIs pharmacokinetic liabilities or secondary mutations that affect the kinase domain of ALK; nevertheless, secondary resistance mutations appear to be the major resistance mechanisms adopted by cancer cells and most importantly the only presently targetable one ${ }^{[3,343]}$.

\section{Secondary resistance mutations}

Considering one of the largest and most extensive systematic analysis addressing ALK inhibitors resistance published by Gainor et al ${ }^{[33]}$ in 2016, assessing 83 repeat biopsies - from as many ALK-positive patients performed from 2009 to 2016 following disease progression on first (crizotinib) and/or second (ceritinib, alectinib, brigatinib) generation ALK-TKIs ( $n=51$ patients received crizotinib, $n=24$ patients received ceritinib, $n=17$ patients received alectinib and $n=6$ patients received brigatinib), ALK secondary resistance mutations were observed in just $20 \%$ of ALK + NSCLC affected patients progressing on crizotinib. On the other hand, the same mutations were detected in 56\% of ALK-rearranged patients progressing on a secondgeneration ALK inhibitor (54\% of patients progressing on ceritinib; $53 \%$ of patients progressing on alectinib and $71 \%$ of patients progressing on brigatinib). G1202R mutation was the most frequent one ${ }^{[33]}$. 
Therefore, treatment with a second-generation ALK inhibitor seems to be directly related to a consistently higher frequency of ALK resistance mutations and to a different range of such ones.

\section{Patients progressing on crizotinib}

ALK secondary resistance mutations were detected in 10 (20\%) patients, L1196M and G1269A being the most common, while other minor detected secondary resistance mutations included: G1202R (2\%), I1171T (2\%), C1156Y (2\%), S1206Y (2\%), and E1210K (2\% $)^{[33]}$.

\section{Patients progressing on ceritinib}

More than half of patients progressing on ceritinib $(13 / 24 ; 54 \%)$ presented ALK secondary resistance mutations, G1202R (21\%) and F1174C/L (16.7\%) being the most frequent ones, moreover, C1156Y mutations were found in the specimens coming from two patients $(8 \%)^{[33]}$.

\section{Patients progressing on alectinib}

Among 17 repeat biopsies performed on patients progressing on alectinib, ALK secondary resistance mutations were observed in $9(53 \%)$ specimens, the most common secondary resistance mutation was G1202R, that was observed in $29 \%$ of cases, while other marginally detected mutations included I1171T/S (12\%), L1196M (6\%) and V1180L (6\% ${ }^{[33]}$.

\section{Patients progressing on brigatinib}

ALK secondary resistance mutations were found in 5 of 7 (71\%) specimens from patients progressing on brigatinib. Once again, just like patients progressing on alectinib or ceritinib, G1202R was the most frequently found ALK secondary resistance mutation, in fact, it was observed in three patients out of seven $^{[33]}$.

\section{Impact of ALK secondary resistance mutations}

Therefore, taking these findings into account, while the main mechanism behind acquired resistance to crizotinib (first generation ALK-TKI) seems to be TKIs pharmacokinetic liability (i.e., resistance occurring due to continued daily therapy over a long period of time), the development of secondary resistance mutations (G1202R being the most prominent one) appears to be the primary mechanism accountable for resistance to and progression after second generation ALK-TKI (ceritinib, alectinib, brigatinib), seemingly due to the increased selectivity and potency of these drugs ${ }^{[35,36]}$.

\section{Bypass signaling tracks}

In contrast with secondary resistance mutations, the activation of bypass signaling pathways represents an "off target" resistance mechanism, meaning that tumor cells manage to exploit other RTKs and/or downstream molecules pathways to overcome ALK dependency in order to keep proliferating despite ALK inhibition. In fact, this activation can involve transmembrane receptors - most notably EGFR and human epidermal growth factor receptor family members - as well as downstream molecules: STAT3, TP53, PIK3CA mutations, MET (MET proto-oncogene, receptor tyrosine kinase) amplification or mitogen-activated protein kinase reactivation, for example ${ }^{[37-39]}$.

To date, none of these bypass pathways is targetable.

\section{FUTURE DIRECTIONS}

The correct employment of genomic information is key to develop even further personalized precision cancer therapies.

In fact, third generation ALK-TKI are currently being specifically developed in order to overcome secondary resistance mutations and more specifically to be effective against G1202R [Table 1]; for example, lorlatinib, 
Table 1. Tyrosine kinase inhibitors effectiveness against secondary resistance mutations

\begin{tabular}{ll}
\hline TKI & Effectiveness against secondary resistance mutations \\
\hline Crizotinib & G1202R: IC50 $=381.6 \mathrm{nmol} / \mathrm{L}$ \\
& L1196M: IC50 $=339.0 \mathrm{nmol} / \mathrm{L}$ \\
& F1174C: IC50 $=115.0 \mathrm{nmol} / \mathrm{L}$ \\
& I1171T/S: IC50 $=51.4 / 94.1 \mathrm{nmol} / \mathrm{L}$ \\
& {$[33]$} \\
& G1202R: IC50 $=124.4 \mathrm{nmol} / \mathrm{L}$ \\
& L1196M: IC50 $=9.3 \mathrm{nmol} / \mathrm{L}$ \\
& F1174C: IC50 $=38.0 \mathrm{nmol} / \mathrm{L}$ \\
Ceritinib & I1171T/S: IC50 $=1.7 / 3.8 \mathrm{nmol} / \mathrm{L}$ \\
& {$[33]$} \\
& G1202R: IC50 $=706.6 \mathrm{nmol} / \mathrm{L}$ \\
& L1196M: IC50 $=117.6 \mathrm{nmol} / \mathrm{L}$ \\
& F1174C: IC50 $=27.0 \mathrm{nmol} / \mathrm{L}$ \\
Alectinib & I1171T/S: IC50 $=33.6 / 117.0 \mathrm{nmol} / \mathrm{L}$ \\
& {$[33]$} \\
& G1202R: IC50 $=129.5 \mathrm{nmol} / \mathrm{L}$ \\
L1196M: IC50 $=26.5 \mathrm{nmol} / \mathrm{L}$ \\
F1174C: IC50 $=18.0 \mathrm{nmol} / \mathrm{L}$ \\
I1171T/S: IC50 $=6.1 / 17.8 \mathrm{nmol} / \mathrm{L}$ \\
{$[33]$}
\end{tabular}

TKI: tyrosine kinase inhibitors; IC: half maximal inhibitory concentration

one of the most promising third generation ALK-TKI, shows activity against all known ALK resistance mutations ( including G1202R: IC50 $=49.9 \mathrm{nmol} / \mathrm{L}$ ), succeeding were first and second generation ALK-TKI failed, as well as excellent results in several different settings: crizotinib-pretreated patients (CNS ORR: 68\%), not crizotinib ALK-TKI pretreated patients (ORR: 33\%, CNS ORR: 42\%), patients pretreated with two or three previous ALK-TKI (ORR: 39\%, CNS ORR: 39\%), naive patients (ORR: 90\%, CNS ORR: 75\%) ${ }^{[33,40]}$.

In the light of the latest ALK-TKI developments, it appears clear that in the near future these drugs will be administered according to a multi-step strategy, based on the information coming from genomic analyses, in order to choose the right drug for the right mutation at the right time, maximizing the benefits coming from the best possible therapeutic sequence.

\section{DECLARATIONS}

\section{Authors' contributions}

All authors contributed equally to the article.

\section{Availability of data and materials}

Not applicable.

\section{Financial support and sponsorship}

None.

\section{Conflicts of interest}

All authors declared that there are no conflicts of interest.

\section{Ethical approval and consent to participate}

Not applicable.

\section{Consent for publication}

Not applicable. 


\section{Copyright}

(c) The Author(s) 2019.

\section{REFERENCES}

1. Pikor LA, Ramnarine VR, Lam S, Lam WL. Genetic alterations defining NSCLC subtypes and their therapeutic implications. Lung Cancer 2013;82:179-89

2. Chia PL, Mitchell P, Dobrovic A, John T. Prevalence and natural history of ALK positive non-small-cell lung cancer and the clinical impact of targeted therapy with ALK inhibitors. Clin Epidemiol 2014;6:423-32.

3. Kim H, Chung JH. Overview of clinicopathologic features of ALK-rearranged lung adenocarcinoma and current diagnostic testing for ALK rearrangement. Transl Lung Cancer Res 2015;4:149-55.

4. Herbst RS, Morgensztern D, Boshoff C. The biology and management of non-small cell lung cancer. Nature 2018;553:446-54.

5. Dela Cruz CS, Tanoue LT, Matthay RA. Lung cancer: epidemiology, etiology, and prevention. Clin Chest Med 2011;32:605-44.

6. Cheng TY, Cramb SM, Baade PD, Youlden DR, Nwogu C, et al. The international epidemiology of lung cancer: latest trends, disparities, and tumor characteristics. J Thorac Oncol 2016;11:1653-71.

7. Holla VR, Elamin YY, Bailey AM, Johnson AM, Litzenburger BC, et al. ALK: a tyrosine kinase target for cancer therapy. Cold Spring Harb Mol Case Stud 2017;3:a001115.

8. Webb TR, Slavish J, George RE, Look AT, Xue L, et al. Anaplastic lymphoma kinase: role in cancer pathogenesis and small-molecule inhibitor development for therapy. Expert Rev Anticancer Ther 2009;9:331-56.

9. Iwahara T, Fujimoto J, Wen D, Cupples R, Bucay N, et al. Molecular characterization of ALK, a receptor tyrosine kinase expressed specifically in the nervous system. Oncogene 1997;14:439-49.

10. Soda M, Choi YL, Enomoto M, Takada S, Yamashita Y, et al. Identification of the transforming EML4-ALK fusion gene in non-small-cell lung cancer. Nature 2007;448:561-6.

11. Hallberg B, Palmer RH. The role of the ALK receptor in cancer biology. Ann Oncol 2016;27 Suppl 3:iii4-15.

12. Ou SI, Shirai K. Anaplastic lymphoma kinase (ALK) signaling in lung cancer. Adv Exp Med Biol 2016;893:179-87.

13. Iyevleva AG, Raskin GA, Tiurin VI, Sokolenko AP, Mitiushkina NV, et al. Novel ALK fusion partners in lung cancer. Cancer Lett 2015;362:116-21.

14. Sabir SR, Yeoh S, Jackson G, Bayliss R. EML4-ALK variants: biological and molecular properties, and the implications for patients. Cancers (Basel) 2017;9:118.

15. Christopoulos P, Endris V, Bozorgmehr F, Elsayed M, Kirchner M, et al. EML4-ALK fusion variant V3 is a high-risk feature conferring accelerated metastatic spread, early treatment failure and worse overall survival in ALK + non-small cell lung cancer. Int J Cancer 2018;142:2589-98

16. Shaw AT, Solomon B. Anaplastic lymphoma kinase (ALK) fusion oncogene positive non-small cell lung cancer. Available from: https:// www.uptodate.com/contents/anaplastic-lymphoma-kinase-alk-fusion-oncogene-positive-non-small-cell-lung-cancer. [Last accessed on 16 Jan 2019]

17. Wu YC, Chang IC, Wang CL, Chen TD, Chen YT, et al. Comparison of IHC, FISH and RT-PCR methods for detection of ALK rearrangements in 312 non-small cell lung cancer patients in Taiwan. PLoS One 2013;8:e70839.

18. Letovanec I, Finn S, Zygoura P, Smyth P, Soltermann A, et al. Evaluation of NGS and RT-PCR methods for ALK rearrangement in European NSCLC patients: results from the European thoracic oncology platform lungscape project. J Thorac Oncol 2018;13:413-25.

19. Hanna N, Johnson D, Temin S, Baker S Jr, Brahmer J, et al. Systemic therapy for stage IV non-small-cell lung cancer: American society of clinical oncology clinical practice guideline update. J Clin Oncol 2017;35:3484-515.

20. Masters GA, Temin S, Azzoli CG, Giaccone G, Baker S Jr, et al. Systemic therapy for stage IV non-small-cell lung cancer: American society of clinical oncology clinical practice guideline update. J Clin Oncol 2015;33:3488-515.

21. Novello S, Barlesi F, Califano R, Cufer T, Ekman S, et al. Metastatic non-small-cell lung cancer: ESMO clinical practice guidelines for diagnosis, treatment and follow-up. Ann Oncol 2016;27:v1-27.

22. ChenY, Fu L. Mechanisms of acquired resistance to tyrosine kinase inhibitors. Acta Pharmaceutica Sinica B 2011;1:197-207.

23. Remon J, Besse B. Brain metastases in oncogene-addicted non-small cell lung cancer patients: incidence and treatment. Front Oncol 2018;8:88.

24. Rocco D, Battiloro C, Della Gravara L, Gridelli C. Safety and tolerability of anaplastic lymphoma kinase inhibitors in non-small-cell lung cancer. Drug Saf 2018; doi: 10.1007/s40264-018-0771-y.

25. Kazandjian D, Blumenthal GM, Chen HY, He K, Patel M, et al. FDA approval summary: crizotinib for the treatment of metastatic nonsmall cell lung cancer with anaplastic lymphoma kinase rearrangements. Oncologist 2014;19:e5-11.

26. Solomon BJ, Mok T, Kim DW, Wu YL, Nakagawa K, et al. First-line crizotinib versus chemotherapy in ALK-positive lung cancer. N Engl J Med 2014;371:2167-77.

27. Solomon BJ, Kim DW, Wu YL, Nakagawa K, Mekhail T, et al. Final overall survival analysis from a study comparing first-line crizotinib versus chemotherapy in alk-mutation-positive non-small-cell lung cancer. J Clin Oncol 2018;36:2251-8.

28. Soria JC, Tan DSW, Chiari R, Wu YL, Paz-Ares L, et al. First-line ceritinib versus platinum-based chemotherapy in advanced ALKrearranged non-small-cell lung cancer (ASCEND-4): a randomised, open-label, phase 3 study. The Lancet 2017;389:917-29.

29. Camidge RD, Peters S, Mok T, Gadgeel SM, Cheema PK, et al. Updated efficacy and safety data from the global phase III ALEX study of alectinib (ALC) vs crizotinib (CZ) in untreated advanced ALK + NSCLC. J Clin Oncol 2018;36:9043. 
30. Khozin S, Blumenthal GM, Zhang L, Tang S, Brower M, et al. FDA approval: ceritinib for the treatment of metastatic anaplastic lymphoma kinase-positive non-small cell lung cancer. Clin Cancer Res 2015;21:2436-9.

31. Larkins E, Blumenthal GM, Chen H, He K, Agarwal R, et al. FDA approval: alectinib for the treatment of metastatic, ALK-positive nonsmall cell lung cancer following crizotinib. Clin Cancer Res 2016;22:5171-6.

32. Kim DW, Tiseo M, Ahn MJ, Reckamp KL, Hansen KH, et al. Brigatinib in patients with crizotinib-refractory anaplastic lymphoma kinasepositive non-small-cell lung cancer: a randomized, multicenter phase II trial. J Clin Oncol 2017;35:2490-8.

33. Gainor JF, Dardaei L, Yoda S, Friboulet L, Leshchiner I, et al. Molecular mechanisms of resistance to first- and second-generation ALK inhibitors in ALK-rearranged lung cancer. Cancer Discov 2016;6:1118-33.

34. Costa DB. Resistance to ALK inhibitors: pharmacokinetics, mutations or bypass signaling? Cell Cycle 2017;16:19-20.

35. Song Z, Wang M, Zhang A. Alectinib: a novel second generation anaplastic lymphoma kinase (ALK) inhibitor for overcoming clinicallyacquired resistance. Acta Pharm Sin B 2015;5:34-7.

36. Rothenstein JM, Chooback N. ALK inhibitors, resistance development, clinical trials. Curr Oncol 2018;25:S59-67.

37. Katayama R. Drug resistance in anaplastic lymphoma kinase-rearranged lung cancer. Cancer Sci 2018;109:572-80.

38. Lin JJ, Riely GJ, Shaw AT, et al. Targeting ALK: precision medicine takes on drug resistance. Cancer Discov 2017;7:137-55.

39. Gainor JF, Dardaei L, Yoda S, Friboulet L, Leshchiner I, et al. Molecular mechanisms of resistance to first- and second-generation ALK inhibitors in ALK-rearranged lung cancer. Cancer Discov 2016;6:1118-33.

40. Shaw AT, Felip E, Bauer TM, Besse B, Navarro A, et al. Lorlatinib in non-small-cell lung cancer with ALK or ROS1 rearrangement: an international, multicentre, open-label, single-arm first-in-man phase 1 trial. Lancet Oncol 2017;18:1590-9. 